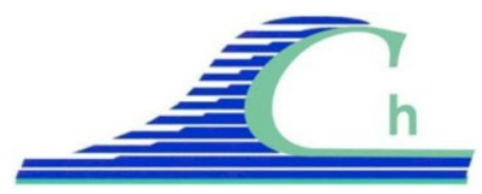

XII ${ }^{\text {èmes }}$ Journées Nationales Génie Côtier - Génie Civil

Cherbourg, 12-14 juin 2012

DOI:10.5150/jngcgc.2012.062-D（C) Editions Paralia CFL

disponible en ligne - http://www.paralia.fr - available online

\title{
Synergie de l'imagerie satellitaire optique et radar pour la cartographie des habitats du Bassin d'Arcachon
}

\author{
Aurélie DEHOUCK ${ }^{1}$, Virginie LAFON $^{1}$, Nicolas BAGHDADI ${ }^{2}$, \\ Vincent MARIEU $^{3,4}$, Bertrand LUBAC ${ }^{3,4}$, Stéphane KERVELLA ${ }^{1}$
}

1. GEO-Transfert, UMR EPOC, avenue des facultés, 33405 Talence, France.

a.dehouck@epoc.u-bordeaux1.fr

2. Irstea, UMR TETIS, 500 rue J.-F. Breton, BP 5095, 34196 Montpellier, France.

3. Université de Bordeaux, UMR EPOC, avenue des facultés, 33405 Talence, France.

4. CNRS, UMR EPOC, avenue des facultés, 33405 Talence, France.

\section{Résumé :}

L'imagerie satellitaire optique dispose d'un bon potentiel de cartographie des espaces naturels littoraux de par l'emprise spatiale, la haute résolution et le recul temporel qu'offrent les 25 ans d'archives image SPOT. Cet article explore le potentiel des images SAR (Synthetic Aperture Radar) Haute Résolution (HR) pour la cartographie des habitats intertidaux en complément de l'imagerie optique traditionnelle. Dans un premier temps, l'article évalue le potentiel de discrimination des habitats du Bassin d'Arcachon à partir d'une série temporelle constituée de 15 images TerraSAR-X et de 2 images ALOS-PALSAR acquises entre 2007 et 2011. L'étude a permis de caractériser les signatures radar de la couverture sédimentaire intertidale, des herbiers de zostères, des bancs d'huitres et de la végétation du schorre. Les bancs d'huîtres, les structures ostréicoles et le schorre ont des signatures radar très particulières (forte brillance) ce qui permet de les distinguer de la slikke, qu'elle soit végétalisée ou non, et de lever la confusion issue de l'analyse d'une image optique seule. Dans un second temps, l'article développe les résultats issus de la classification des données optiques et radar concaténées sur le secteur de La Hume. Les résultats les plus satisfaisants sont obtenus avec une classification supervisée de Mahalanobis basée sur les 4 bandes SPOT d'une image estivale, la bande XS2 d'une image SPOT d'hiver et une bande radar HH TerraSAR-X. Cette approche cartographique combinant données optiques-radar multitemporelles permet de lever les principales difficultés ayant motivé cette étude. Des développements sont toutefois encore nécessaires pour parvenir à mieux cartographier les grands ensembles végétaux du schorre et à détecter les biofilms à microphytobenthos ainsi que les dépôts de macro-algues.

Mots-clés :

Télédétection - SPOT - TerraSAR-X - ALOS-PALSAR - Cartographie - Habitats Littoral 


\section{Introduction}

De nombreux travaux ont démontré le potentiel des images de télédétection spatiale pour la cartographie des habitats naturels des zones côtières. Le Bassin d'Arcachon, lagune macrotidale du sud-ouest de la côte Atlantique, site Natura 2000 et futur parc marin, est un site de démonstration privilégié où les écosystèmes doivent s'adapter au changement du climat et aux usages anthropiques. Dès lors, la télédétection spatiale est un outil efficace et bon-marché pour cartographier les habitats naturels du Bassin et suivre leurs évolutions spatio-temporelles. Des travaux précédents ont démontré le potentiel des images de télédétection spatiale haute résolution pour la cartographie des faciès bio-sédimentaires du Bassin (LAFON et al., 2008) et des zones naturelles littorales de par le monde (SANCHEZ-HERNANDEZ et al., 2007 ; SYAMSUL \& AGUS, 2007). Cependant, l'imagerie multispectrale optique de résolution décamétrique (ex : SPOT et Formosat) peine à détecter correctement les bancs d'huitres, les herbiers de zostères peu denses et à discriminer herbiers denses de zostères et végétation du schorre (LAFON et al., 2008). Ces lacunes peuvent en partie être levées en incorporant des données radar HR qui ont un potentiel intéressant d'imagerie des zones intertidales (VAN DER WAL et al., 2005; GADE et al., 2008 ; SLATTON et al., 2008).

L'article vise à confirmer le potentiel des images radar TerraSAR-X et ALOS-PALSAR pour la cartographie des habitats naturels intertidaux en complément de l'imagerie optique traditionnelle. Dans un premier temps, l'apport des données SAR est discuté en décrivant les signatures radar de chacun des habitats et leur séparabilité radiométrique. Dans un second temps, les résultats d'une classification supervisée issue des données optiques et radar concaténées sont présentés démontrant l'apport significatif des images radar HR pour l'amélioration des cartographies des habitats intertidaux. Nous nous limiterons ici à un site test situé au sud du Bassin d'Arcachon (site de la Hume, d'emprise $1.8 \mathrm{~km} \times 2.3 \mathrm{~km}$ ) régulièrement suivi sur le terrain et qui représente la diversité des habitats rencontrés dans le Bassin d'Arcachon.

\section{Matériel et méthodes}

\subsection{Données satellitaires}

Les images satellite exploitées dans ces travaux sont issues de la base de données Kalideos Littoral (CNES, http://www.kalideos.cnes.fr) qui rassemble plus de 150 images optiques et radar HR du Bassin d'Arcachon. Dans le cadre du programme SYNIHAL, 15 images TerraSAR-X (bande X, $9.65 \mathrm{GHz}$, longueur d'onde $3 \mathrm{~cm}$ ) sont examinées, acquises en double polarisation HH-VV, HH-HV ou VV-VH, en mode Stripmap $(2.75 \mathrm{~m})$ ou Spotlight $(1$ à $1.5 \mathrm{~m})$. Les images TerraSAR-X ont été acquises dans des conditions instrumentales variées (polarisation, angle d'incidence, angle de prise de vue radar selon orbite ascendante/descendante). Parmi elles, quatre images n'ont pas été exploitées, trop bruitées radiométriquement car acquises pour des angles 


\section{XII ${ }^{\text {èmes }}$ Journées Nationales Génie Côtier - Génie Civil \\ Cherbourg, 12-14 juin 2012}

d'incidence extrêmes $\left(15^{\circ}\right.$ et $\left.55^{\circ}\right)$. Deux images ALOS-PALSAR (bande L, $1.27 \mathrm{GHz}$, $24 \mathrm{~cm})$, polarisation double HH-HV $(12.5 \mathrm{~m})$, ont également été acquises. Toutes les images radar acquises correspondent à des niveaux d'eau de basse mer. Les images TerraSAR-X et ALOS-PALSAR sont calibrées en coefficient de rétrodiffusion radar $\left(\sigma_{0}\right.$ en dB) (FRITZ, 2007). Les images optiques SPOT-5 sont livrées en niveau 3, géoréférencées par le CNES en s'appuyant sur une image SPOT-5 $(2.5 \mathrm{~m})$ de référence par reconnaissance de points homologues. Les images Formosat-2, TerraSAR-X et ALOS-PALSAR sont co-géoréférencées sur la base d'une image SPOT-5 $(10 \mathrm{~m})$ de la BD Kalideos pour être superposables les unes aux autres (erreur rms de géoréférencement de l'ordre de 2 pixels TerraSAR-X $\approx 5 \mathrm{~m}$ et 0.3 pixel pour ALOSPALSAR $\approx 4 \mathrm{~m}$ ).

Des données marégraphiques (niveau d'eau réel, coefficient de marée, niveau d'eau atteint lors de la pleine mer précédente) et météorologiques (vent, pluviométrie) décrivent les conditions environnementales précédant et pendant les acquisitions radar.

\subsection{Données de terrain}

Des missions d'observation synchrones à l'acquisition des images radar sont utilisées pour décrire l'état des surfaces et en interpréter leur signature radar. Ces données ne sont pas présentées dans cet article. Nous disposons de photographies numériques géoréférencées (description de la nature du substrat), d'observations qualitatives et de mesures quantitatives des propriétés des sols notamment de leur rugosité (caractéristiques des champs de rides sableuses, des vasières, de la rugosité des herbiers de zostères) et de leur humidité (description qualitative de l'état d'humidité et d'inondation des sols, mesure quantitative de la teneur en eau). Ces observations sont accompagnées de leur positionnement GPS assuré avec un Trimble GEO XT de précision métrique. Ces observations de terrain sont indispensables pour une bonne interprétation des images radar au vu de la forte variabilité spatio-temporelle des figures sédimentaires, des conditions d'humidité et d'inondation induites par la marée et l'agitation des vagues. Un survol aérien (prises de vue verticales à $40 \mathrm{~cm}$ de résolution) opéré par la société l'Avion Jaune en mars 2011 aide également à l'interprétation des signatures radar des habitats.

Outre ces observations, la récurrence des missions hyperspectrales réalisées en 2010 et 2011 environ tous les 2 mois permet d'avoir une bonne connaissance des évolutions saisonnières des états de surface notamment au regard du développement de l'herbier de zostères et de l'occurrence de blooms d'algues vertes. Les mesures optiques sont réalisées à l'aide de deux spectro-radiomètres TRIOS RAMSES qui permettent de restituer pour chaque type de surface, sa réflectance de télédétection. Parmi les autres données utilisées, citons le cadastre ostréicole fourni par la DDTM33-Arcachon ainsi que les couches SIG "bancs d'huitres" (méthodologie décrite dans LAFON et al., 2010) 
et "front du schorre" (photo-interprétation) issus du traitement d'une orthophoto 2007 du Bassin d'Arcachon dans le cadre de l'inventaire biologique Natura 2000 en Mer.

\subsection{Stratégie de cartographie}

Plusieurs couples d'images optiques/SAR sont éligibles pour cartographier les habitats intertidaux et supratidaux. Les données TerraSAR-X sont rééchantillonnées à $10 \mathrm{~m}$ (résolution des images SPOT-5) par la technique d'agrégation de pixels qui permet de lisser la variabilité inter-pixellaire et ainsi d'atténuer le speckle. L'ensemble des bandes optiques et SAR retenues sont concaténées et classées à l'aide de trois algorithmes supervisés : minimum de distance, Mahalanobis et maximum de vraisemblance. 21 classes d'entrainement ont été définies à partir des observations in situ et des signatures radiométriques optiques et radar des substrats afin de représenter la diversité des substrats. Différentes combinaisons de bandes spectrales optiques et radar sont testées.

\section{Résultats et discussion}

\subsection{Signature optique des habitats}

Les mesures optiques estivales de terrain montrent une bonne séparabilité spectrale entre les sols couverts de végétation et les sols nus exceptés pour les plus faibles taux de recouvrement de l'herbier (figure 1).

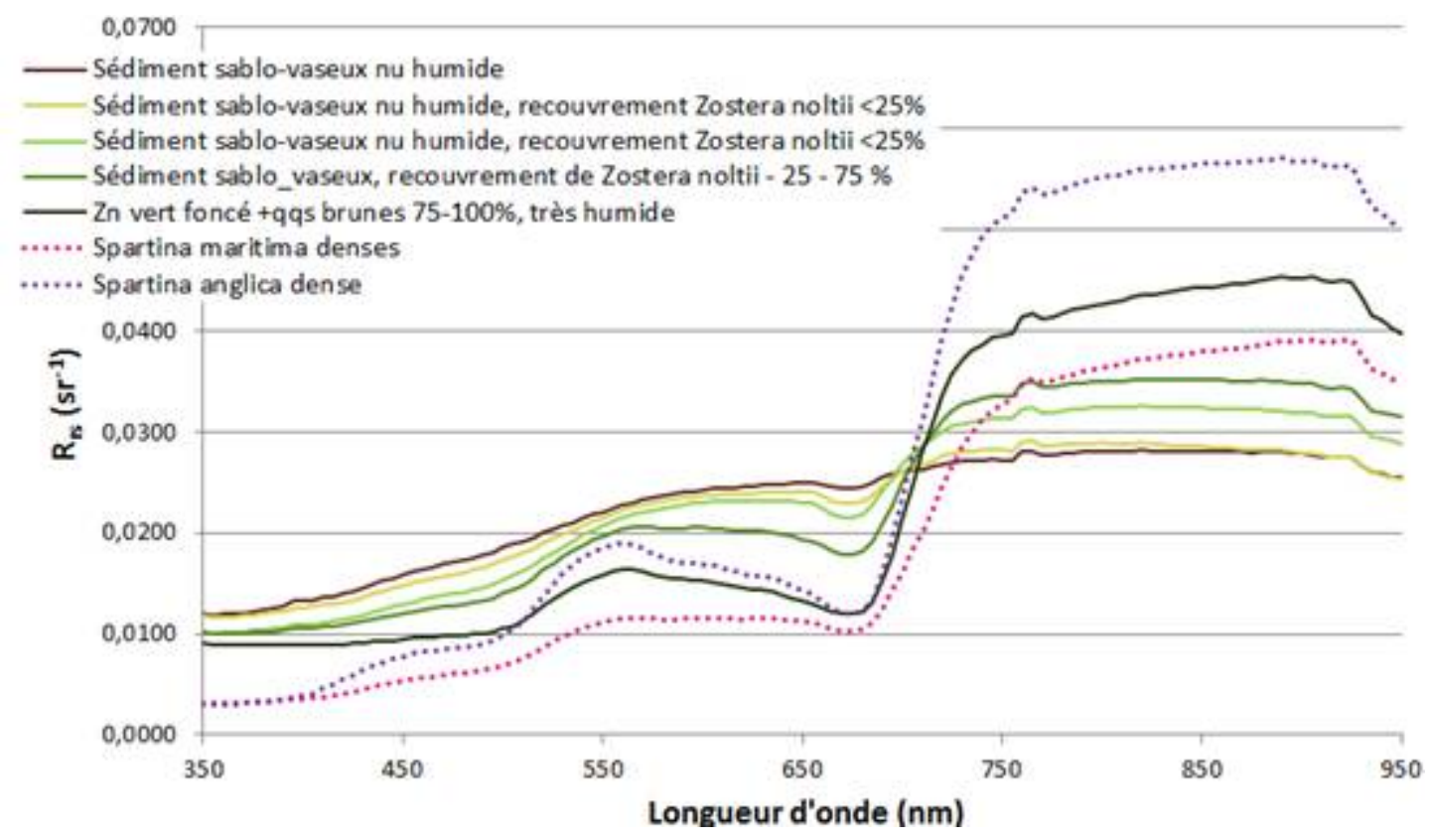

Figure 1. Spectres radiométriques acquis à La Hume le 01/08/2011. 


\section{XII ${ }^{\text {èmes }}$ Journées Nationales Génie Côtier - Génie Civil \\ Cherbourg, 12-14 juin 2012}

Nous remarquons une forte dynamique radiométrique dans les longueurs d'onde rouge $(610-700 \mathrm{~nm})$ et proche infrarouge $(780-900 \mathrm{~nm})$. Dans le rouge, une propriété particulière apparaît : les spartines (limite basse du schorre ; courbe rose) et les zones d'herbiers présentant un fort taux de recouvrement (courbe vert foncé) ont des réflectances dans le rouge très faibles et très proches. Au contraire, les sols nus (courbe marron) et à faible et moyen taux de recouvrement de zostères (courbes vert clair) présentent des radiométries plus fortes et plus variables. Or, en hiver, les taux de recouvrement de l'herbier sont généralement faibles à l'échelle du Bassin (AUBY \& LABOURG, 1996). Le canal rouge peut donc être discriminant vis-à-vis des différentes espèces dans la perspective d'une combinaison multi-temporelle. Les couverts d'huitres, recouverts de micro-algues, répondent comme de la végétation. Ils n'ont donc pas une signature optique typique.

\subsection{Signature SAR des habitats}

L'analyse des données SAR indique un fort potentiel des images radar ALOS-PALSAR et TerraSAR-X pour combler les lacunes issues de l'utilisation d'images optiques seules pour la cartographie des habitats intertidaux. En premier lieu, les bancs d'huitres sont facilement discriminables des autres substrats, nus ou végétalisés par les zostères, aussi bien en bande X qu'en bande L (forte brillance des bancs d'huitres sur la figure 2). La différence est de 4 à $8 \mathrm{~dB}$ en polarisation $\mathrm{HH}(>2 \mathrm{~dB}$, seuil de séparabilité de deux faciès dérivé de la précision radiométrique des images SAR) entre les bancs d'huitres et les sols voisins en considérant l'ensemble des données TerraSAR-X. Ceci s'explique par la rugosité centimétrique des coquilles d'huitres responsable d'une réflexion diffuse et conduisant à une forte rétrodiffusion radar en bande $X\left(\sigma_{0, н н} \approx-7 \mathrm{~dB}\right.$ à $\left.-9 \mathrm{~dB}\right)$ ainsi qu'en bande $\mathrm{L}\left(\sigma_{0, \mathrm{HH}}=-12 \mathrm{~dB}\right)$. Les images radar apportent aussi une contribution majeure pour améliorer la séparabilité entre l'herbier de zostères et le schorre. La différence est d'environ $4 \mathrm{~dB}$ en bande $\mathrm{L}$ et varie entre 2 et $7 \mathrm{~dB}$ en bande $\mathrm{X}$. Le schorre est caractérisé par une très forte rétrodiffusion radar du fait de mécanismes de diffusion double et multiple (interactions sol-plante et eau-plante) et par l'humidité des sols du fait de l'inondation lors de la pleine mer précédente. Sur toutes les images SAR, on observe un gradient cross-shore de la réponse radar du bas schorre (très brillant) vers le haut schorre (sombre) qui est corrélé à l'humidité et indirectement à la nature des sols (sols vaseux peu drainants restant très humides sur le bas schorre et le schorre moyen, sol sableux très drainant s'asséchant vite sur le haut schorre). D'une manière générale, il existe de fortes variations de la réponse radar associées à une variabilité spatiotemporelle des propriétés de surface des sédiments (présence/disparition de figures sédimentaires, conditions d'humidité du sol et d'inondation par la marée variables) ainsi qu'à des facteurs instrumentaux ( $\sigma_{0}$ décroît avec un angle d'incidence croissant, $\sigma_{0}$ des rides sableuses influencé par l'angle de visée du satellite). 


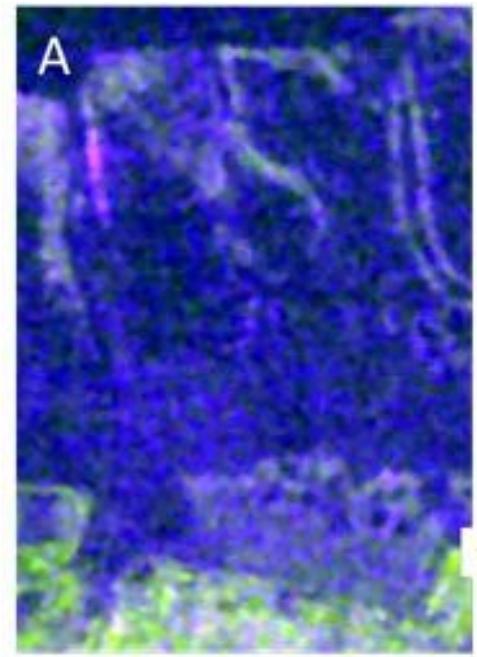

ALOS $5 / 10 / 09 \mathrm{HH}_{\mathrm{H}} \mathrm{HV}\left(39^{*}\right)$

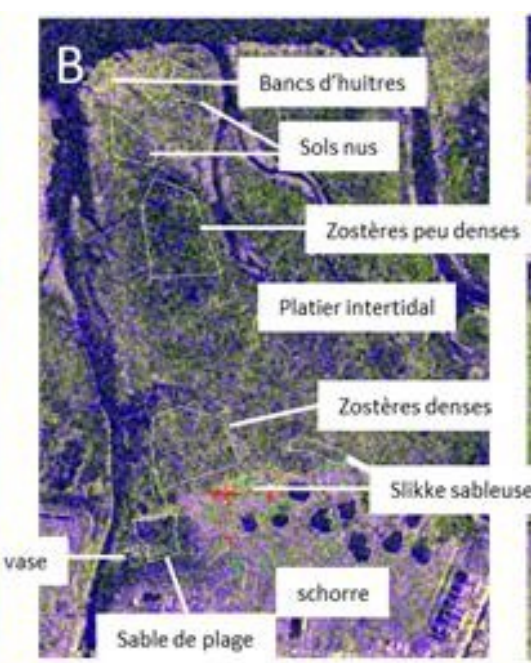

TSX 11/12/09 HH,W(33", D)

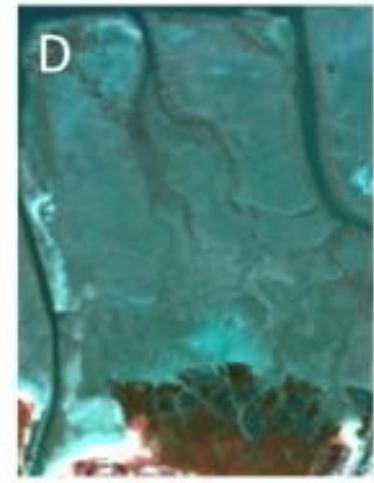

SPOT-5 01/02/10

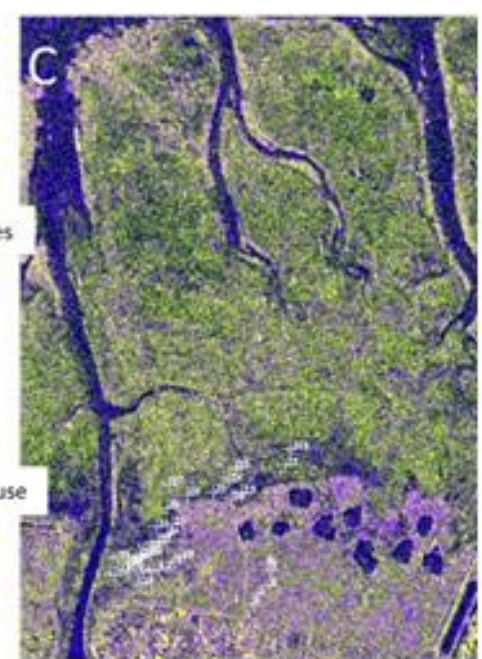

TSX 07/09/11 HH,W(34; , A)

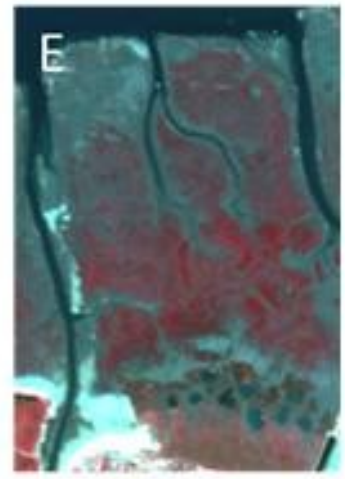

SPOT-5 28/8/11

Figure 2. Images ALOS-PALSAR du 5/10/09 (A), TerraSAR-X des 11/12/09 et 07/09/11 (B-C), SPOT-5 des 01/02/10 et 28/08/11 (D-E).

\section{$\underline{3.3 \text { Classification optique - SAR }}$}

Un couple constitué d'une image satellite optique (SPOT-5 à $10 \mathrm{~m}$ du 28/08/11) et d'une image radar (TerraSAR-X à $2.75 \mathrm{~m}$ du 7/09/11) a été retenu du fait de la proximité des deux acquisitions et de leur niveau d'eau semblable. En outre, ce couple d'images estivales permet de décrire l'herbier de zostères au plus fort de son développement (AUBY \& LABOURG, 1996). La bande rouge extraite de la scène SPOT-5 du 01/02/2011 a également été sélectionnée afin d'en analyser l'utilité dans la perspective de la cartographie thématique (figure 3). Cinq bandes optiques SPOT-5 (canaux Vert, Rouge, PIR, MIR de l'été et Rouge de l'hiver) ainsi que les 2 bandes TerraSAR-X $(\mathrm{HH}, \mathrm{VV})$ à $10 \mathrm{~m}$ de résolution sont concaténées en une seule image de 7 canaux.

Parmi les différents tests de classification, la méthode de Mahalanobis donne les résultats les plus satisfaisants. La figure $3 \mathrm{~b}$ montre les résultats de la classification de 


\section{XII ${ }^{\text {èmes }}$ Journées Nationales Génie Côtier - Génie Civil \\ Cherbourg, 12-14 juin 2012}

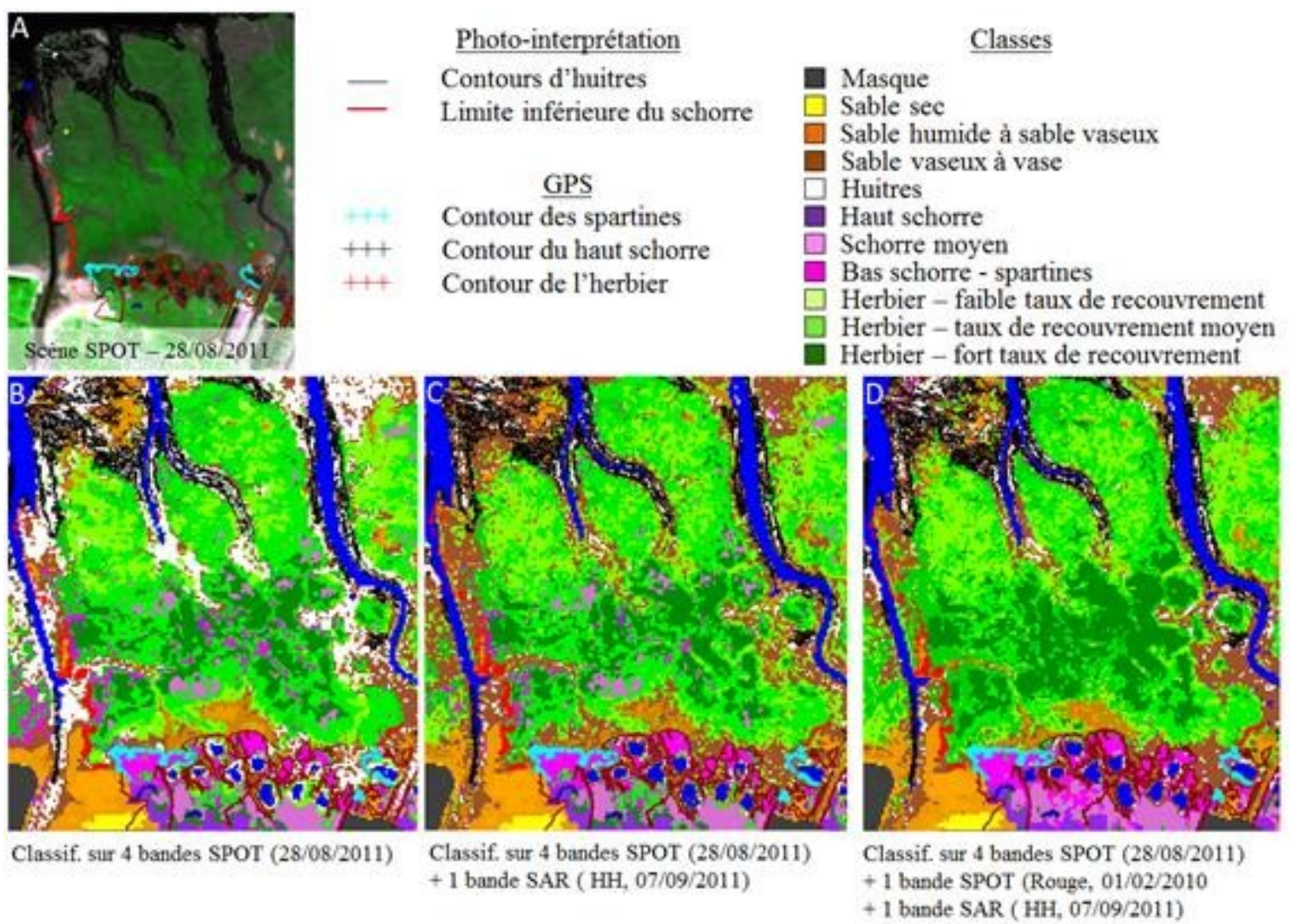

Figure 3. Résultats des tests de classification (B-D) sur les images SPOT-5 (28/8/11 et 1/02/10) - TerraSAR-X (7/9/11). L'image SPOT (A) montre les classes d'entrainement utilisées et les données de terrain (contours et points) qui sont également superposées

aux cartographies (B-D).

l'image SPOT du 28/8/11. On note une sur-détection des bancs d'huitres (grandes étendues blanches débordant des contours noirs obtenus par photo-interprétation) ainsi que de nombreux patchs de végétation du schorre (rose/violet) au sein de l'herbier (vert), et réciproquement. L'intégration d'une bande radar permet de résoudre les problèmes de sur-détection des huitres et de réduire la confusion entre l'herbier et le schorre (figure 3c). En incorporant la bande rouge SPOT-5 d'hiver (1/02/10), la confusion herbier/schorre est presque totalement levée (figure $3 \mathrm{~d}$ ).

Ces résultats sont très encourageants et indiquent que l'utilisation combinée d'images multi-temporelles optiques et radar permet de résoudre les principales difficultés issues de l'utilisation d'une image optique seule. Cependant, des améliorations sont encore à apporter. Tout d'abord, les données de terrain indiquent que les grandes ceintures végétales du schorre ne sont pas encore bien discriminées. Des mesures optiques complémentaires doivent être réalisées pour mieux comprendre les signatures saisonnières des grands ensembles du schorre afin de les exploiter dans une approche de classification multi-temporelle mieux adaptée. En outre, rappelons que la signature radar des prés-salés est très influencée par l'humidité du sol, résultant de l'inondation 
par la marée, associée à la nature du substrat. En exploitant plus avant ce marqueur dans une approche combinée optique-radar, nous pouvons espérer améliorer la cartographie du moyen schorre et du haut schorre. Nous constatons aussi que l'herbier s'étend audelà des contours levés sur le terrain (croix rouges sur la figure 3). Il s'agit vraisemblablement de dépôt d'algues ou de biofilms sujets à une grande variabilité spatiale et temporelle. Par manque d'observations géoréférencées au moment des acquisitions, nous n'avons pas pu intégrer des parcelles d'entrainement qui leur seraient dédiées.

\section{Conclusions}

L'analyse des données radar TerraSAR-X et ALOS-PALSAR acquises sur le Bassin d'Arcachon confirme le potentiel des images SAR pour améliorer la performance de la cartographie des habitats naturels en complément des images optiques. Les bancs d'huitres et le schorre ont une signature radar prononcée ce qui permet de les discriminer des autres surfaces intertidales. Des combinaisons de bandes spectrales optiques et radar ont été testées. La meilleure classification est obtenue en utilisant un algorithme supervisé de Mahalanobis sur une image composée de 4 bandes SPOT-5 d'une scène estivale $(28 / 8 / 11)$, de la bande XS2 d'une scène SPOT-5 hivernale $(1 / 02 / 10)$ et de la bande radar HH TerraSAR-X (7/09/11). La confrontation des résultats aux données de terrain indique une détection parfaite des bancs d'huitres par l'introduction de la bande radar $\mathrm{HH}$ et une bonne discrimination herbier/schorre par l'assimilation conjointe de la bande SPOT XS2 d'hiver et des données radar. Toutefois, des efforts sont encore nécessaires pour parvenir à détecter les macro-algues et les couverts sédimentaires à microphytobenthos dans l'optique d'aboutir à une cartographie thématique exhaustive des habitats du Bassin d'Arcachon.

\section{Remerciements}

Les auteurs remercient le CNES (Centre National d'Etudes Spatiales) pour son soutien et le financement du programme SYNIHAL (TOSCA 2011). Les images satellitaires proviennent de la base de données KALIDEOS du CNES. Les images TerraSAR-X ont été obtenues dans le cadre des propositions LAN237 et COA1163 du DLR.

\section{Références bibliographiques}

AUBY I., LABOURG P.-J. (1996). Seasonal dynamics of Zostera noltii Hornem. in the bay of Arcachon (France). Journal of Sea Research, 35 (4), pp 269-277. doi:10.1016/S13851101(96)90754-6

FRITZ (2007). TerraSAR-X Ground Segment Level $1 b$ Product Format Specification. Report TX-GS-DD-3307, Issue 1.3, pp 257.

LAFON V., MARIEU V., BUTEL R., DEHOUCK A., FROIDEFOND J.M., TRUT G. (2008). Cartographie des faciès bio-sédimentaires du Bassin d'Arcachon à partir de 


\section{XII ${ }^{\text {èmes }}$ Journées Nationales Génie Côtier - Génie Civil \\ Cherbourg, 12-14 juin 2012}

l'imagerie FORMOSAT-2. 10èmes Journées Nationales Génie Côtier Génie Civil, 14-16 octobre 2008, Sofia Antipolis, pp 563-572. doi:10.5150/jngcgc.2008.054-L

LAFON V., MUGICA J., MALlET C. (2010). Cartographie de l'état du Domaine Public Maritime par télédétection THR: Application au Bassin d'Arcachon. XIème Journées Nationales Génie Côtier Génie Civil, Les Sables d'Olonne, 22-25 juin 2010, pp 505-512. doi:10.5150/jngcgc.2010.059-L

GADE M., ALPERS W., MELSHEIMER C., TANCK G. (2008). Classification of sediments on exposed tidal flats in the German Bight using multi-frequency radar data. Remote Sensing of Environment, 112, pp 1603-1613. doi:10.1016/j.rse.2007.08.015

SANCHEZ-HERNANDEZ C., BOYD D.S., FOODY G.M. (2007). Mapping specific habitats from remotely sensed imagery: Support vector machine and support vector data description based classification of coastal saltmarsh habitats. Ecological Informatics, 2(2), pp 83-88. doi:10.1016/j.ecoinf.2007.04.003

SYAMSUL M., AGUS B. (2007). Formosat-2 satellite imagery assessment for coastal ecosystem mapping in western coast of Banten, Indonesia. Proceedings of SAKE-2 Workshop.

SLATTON K.C., CRAWFORD M.M., CHANG L.D. (2008). Modeling temporal variations in multipolarized radar scattering from intertidal coastal wetlands. ISPRS Journal of Photogrammetry and Remote Sensing, 63, pp 559-577. doi:10.1016/j.isprsjprs.2008.07.003

VAN DER WAL D., HERMAN P., WIELEMAKER-VAN DEN DOOL A. (2005). Characterisation of surface roughness and sediment texture of intertidal flats using ERS SAR imagery. Remote Sensing of Environment, 98, pp 96-109. doi:10.1016/j.rse.2005.06.004 
Thème 3 - Instrumentation, mesures, imagerie et télédétection 